5 Stoner C E Jr, Haggerty B S. Thermal decomposition of energetic materials55. metal complex es of Diaminoglyoxime as potential burn rate modifiers in composite propellants. Propellants, Explosives, Pyrotechnics, 1992, 17: 82 89

6 Grigull U, Staub J, Schiebener P. Steam Tables in SI_U nits. 3rd ed. New York: Springer_Verlag, 1990. 57

\title{
膜结合型巨噬细胞集落刺激 因子的受体作用
}

\section{吴克复 何志鸿 郑国光 饶 青 王 昕} (中国医学科学院, 中国协和医科大学血液学研究所, 实验血液学国家重点实验室, 天津 300020)

摘要 根据细胞因子的定义, 信息应由因子流向受体, 可溶性受体与膜结合因子结合可有信息反向传导. 人 白血病细胞系 J6_1 细胞表面同时存在膜结合型 M_CSF 及其受体, 构成自家并置性刺激. 将由 J6_1 细胞膜分 离的 M_CSF 可溶性受体加入 J6_1 细胞培养观察到细胞增殖受抑制, 分裂指数降低, 细胞直径增大, 多核细胞 比例减少和多种细胞表面抗原表达的下调. J6_1 细胞在可溶性受体作用数分钟至 $20 \mathrm{~min}$ 可观察到细胞内 pH 的明显变化, 表明有信号通过 m_M_CSF 传入细胞, 这种信号传入不受 m_M_CSF 糖基化影响.

\section{关键词 细胞因子 巨噬细胞集落刺激因子 受体 并置性刺激}

细胞因子 ( 以下简称因子) 是细胞间通讯的主要信号分子. 分泌型因子通过内分泌 (Endocrine), 旁分泌(Paracrine) 或自分泌(Autocrine) 机制进行信息传递. 膜结合型因子则通过并 置性刺激( Juxtacrine) 传递信息 ${ }^{[1]}$. 根据因子的定义, 信息由因子流向受体.

人白血病细胞系 J6_1 有明显的成丛生长现象 ${ }^{2]}$, 其增殖和生存依赖于膜结合型巨噬细胞 集落刺激因子及其受体 ( m_M_CSF_M-CSF-R) 介导的自家并置性刺激 ${ }^{[3]}$, 这种机制在部分白 血病患者骨髓细胞和一些肿瘤组织中存在 ${ }^{[4]}$. 在研究由 J6_1 细胞提取的 M_CSF-R 对 J6_1 细 胞的生物学作用中, 观察到了增殖抑制效应 ${ }^{[5]}$, 符合用 M_CSF 被结合后因子减少应有的效果 推测. 但是, 深入研究, 则出现了与经典信息流向相悖的现象, 即有事实表明在此信息是由 $\mathrm{M}_{-}$ CSF-R 流向 m_M_CSF 的, 提示自家并置性刺激可能有更复杂的作用机制.

\section{1 材料与方法}

J6_1 细胞系建于 1976 年, 经 20 多年研究其性质和特点已比较清楚 ${ }^{[2,3,5]}$. 本文使用液氮 保存 100 120 代的细胞, 培养方法如文献[ 2] 所述.

J6_1 细胞 M_CSF_R 的分离如文献 [ 5] 所述, 本文用经 FPLC M ono Q 分离的活性组分作为 实验用 M_CSF-R, 以 $50 \%$ 抑制作为 $1 \mathrm{U}$.

细胞内 $\mathrm{pH}$ 变化测定: $\mathrm{pH}$ 敏感的荧光激活剂 $2^{\prime} 7^{\prime}$-bis ( carboxyethyl) 5(6)_carbo_fluorescein acetoxymethyl ester (BCECF/ AM) 购自 Boehringer M annheim. $5 \times 10^{6} \mathrm{~J} 6 \_1$ 细胞经 Hank 氏液

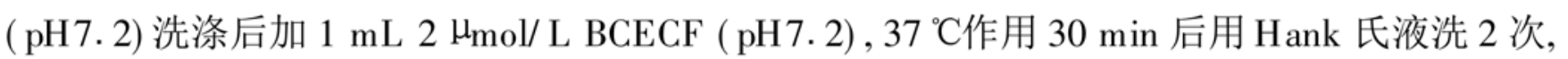
再悬浮于 $5 \mathrm{~mL}$ Hank 氏液中, 置冰浴备用. 各加 $50 \mu \mathrm{L}(0.08 \mathrm{U})$ 或 $100 \mu \mathrm{L}(0.16 \mathrm{U}) \mathrm{M} \_\mathrm{CSF}$ R, 在流式细胞仪(FACS, Couter, Epiocs) 上测定经 M_CSF_R 作用 10, $20 \mathrm{~min}$ 的由 $500 \mathrm{~nm}$ 和 
$450 \mathrm{~nm}$ 激光激发产生的 $530 \mathrm{~nm}$ 苂光强度的比值, 以胎牛血清和 Hank 氏液作为阳性和阴性 对照.

形态学效应观察: J6_1 细胞按 $1 \times 10^{5} / 200 \mu \mathrm{L}$ 孔接种于 96 孔培养板, 每孔加 M_CSF-R $0.4 \mathrm{U} / 40 \mu \mathrm{L}$ 或磷酸盐缓冲液( PBS) $40 \mu \mathrm{L}$ 孔(阴性对照), 每组 4 孔. $5 \mathrm{~min}$ 后取其中各 2 孔 用 Cytospin 2 (Shandon) 作离心涂片; $40 \mathrm{~min}$ 后其余 2 孔各加 $40 \mu \mathrm{L} \mathrm{FCS.} 37^{\circ} \mathrm{C}$ 培养 $24 \mathrm{~h}$ 后用 Cytospin 2 作离心涂片, 所有涂片均作 Giemsa 染色. 测量作用 $5 \mathrm{~min}$ 后的细胞直径和细胞核 直径, 每组各测 100 个单个核细胞, 按下式计算细胞与细胞核的体积比:

$$
V_{\mathrm{d}} / V_{\mathrm{n}}=(\mathrm{dc})^{3} /(\mathrm{dn})^{3} \text {. }
$$

在培养 $24 \mathrm{~h}$ 的细胞离心涂片中计数多核细胞的比例, 各组计数 4000 个细胞.

细胞表面抗原测定: 抗 M_CSF 单抗 ${ }^{[6]}$ 和抗 M-CSF-R 单抗均由我室制备, 抗 Endoglin 单 抗, 抗 HLA-ABC 单抗和抗 HLA-DR 单抗均由德国 T•bingen 大学 Hans B•hring 博士惠赠, ABC 免疫酶标法按我室常规 ${ }^{[7]}$.

神经氨酸酶处理试验: 神经氨酸酶购自 Sigma. 取对数生长期的 J6_1 细胞, 经 RPM I 1640 洗涤后, 按 $1 \times 10^{6} / \mathrm{mL}$ 接种 24 孔板 4 孔, 其中 2 孔加入 $0.01 \mathrm{U}$ 神经氨酸酶. $37{ }^{\circ} \mathrm{C}, 1 \mathrm{~h}$ 后再 洗涤再接种于 24 孔板, 其中 2 孔加 $4.0 \mathrm{U} / \mathrm{mL}$ M_CSF-R(对照 2 孔加入 PBS). 各加 5\% FCS, 培养 $16 \mathrm{~h}$ 时后用 ${ }^{3} \mathrm{H}-\mathrm{TdR}$ 掺入率测出抑制活性(用 Beckman LS5081 液体闪㷧仪测定, 一式 3 份).

\section{2 结果与讨论}

我们过去的工作业已证明 m_M_CSF/ M_CSF_R 是 J6_1 细胞赖以生存的自家并置性刺激 机制 ${ }^{[3]}$ ( 因为 J6_1 细胞培养上清液没有 M_CSF 含量的明显增高, 即 M_CSF 及其受体并不以 一般的自分泌机制起作用).J6_1 细胞在可溶性受体( s_M_CSF_R) 作用下, 表面的 m_M_CSF 与 sM_CSF_R 结合, 自家并置性刺激机制减少, 因而细胞增殖受到抑制. 已用集落试验和裸鼠 接种试验证实 ${ }^{[5]}$; 同理抗 M_CSF 单抗对 J6_1 细胞增殖也有抑制作用 ${ }^{[5]}$.

最近, 我们用由人胎盘克隆的 M_CSF_R cDNA 胞外区 ${ }^{1)}$ 的大肠杆菌表达产物, 即重组的 sM-CSF-R 作实验, 对 J $6-1$ 细胞增殖也有抑制作用, 而用该抑制作用可被抗 M_CSF_R 的单抗 中和( 资料另文发表) . sM_CSF_R 对 J6_1 细胞的抑制作用是否代表完整 J6_1 细胞间 m_M CSF/ M_CSF-R 的作用有待研究, 但抑制效应解释了 J6_1 细胞在成丛生长时增殖不旺盛 ${ }^{[2]}$ 的 部分原因, 也引发了我们对其进行深入研究.

上述抑制是用半固体培养体系作的, 即培养 $4 \mathrm{~d}$ 以上的效应. 本文用液体培养测定了 72 $\mathrm{h}$ 内的生长曲线, sM_CSF_R 对 J6_1 细胞增殖也显示了抑制效应, 培养 $6 \mathrm{~h}$ 与对照组相比抑制 率达 $25.4 \%$, 培养 $24 \mathrm{~h}$ 分裂指数有差异(对照 $22 \%$, 实验 $17 \%, p<0.01$ ), 随着时间的后移抑 制作用减弱, 可能与 sM_CSF_R 一次性加入后降解有关.

用流式细胞仪作的细胞内 $\mathrm{pH}$ 变化表明在 sM_CSF_R 作用下, 数分钟至 20 min J6_1 细胞 的胞内 $\mathrm{pH}$ 有明显的变化(图 1), 表明有信息传入. 同时细胞直径增大: 作用 $5 \mathrm{~min}$ 后实验组 平均直径( 19.5 士3.5) $\mu_{\mathrm{m}}$, 对照组 $(14.6 \pm 2.7) \mu_{\mathrm{m}}, p<0.001$. 细胞体积与核体积比, 实验 组 $3.5 \pm 1.5$, 对照组 $1.6 \pm 0.6, p<0.001$, 表明在信号传入时细胞骨架有明显变化. 在 $\mathrm{sM}_{-}$

1) 王 昕, 蔡辉国, 陈佩贞, 等. 人胎盘 $\mathrm{cfms}$ 胞外功能区基因片段的扩增. 生物化学杂志 (待发表) 
CSF_R 作用下培养 $24 \mathrm{~h}$ 后 J6_1 细胞 多核细胞比例明显减少(图 2(a)), 也 表明细胞骨架有变化. 值得注意的是 抗 M_CSF 单抗处理 J6_1 细胞, 培养 $24 \mathrm{~h}$ 后也有明显的多核细胞减少现象 (图 2(b)). 说明 I6 1 细胞有核细胞, 比 例减少与 m_M_CSF 被结合传入的信 息有关. 同时, 在 sM_CSF_R 作用下 J6_1 细胞表面的 M_CSF, M_CSF_R, HLA_ABC, HLADR 和 Endoglin 抗原 表达也明显下调(图 3), 说明其作用和 影响相当广泛.

${ }^{3} \mathrm{H} \_\mathrm{T} d R$ 掺入试验表明用神经氨酸酶 处理 J6_1 细胞 m_M_CSF 和 M_CSF_R 作用没有明显的影响; 因为实验组和 对照组 ${ }^{3} \mathrm{H}-\mathrm{TdR}$ 掺入率没有明显差异, 揭示糖基在 m_M_CSF 与 M_CSF_R 作 用中不起重要作用. 最近用原核细胞 表达的重组 M_CSF_R 对 J6_1 细胞也

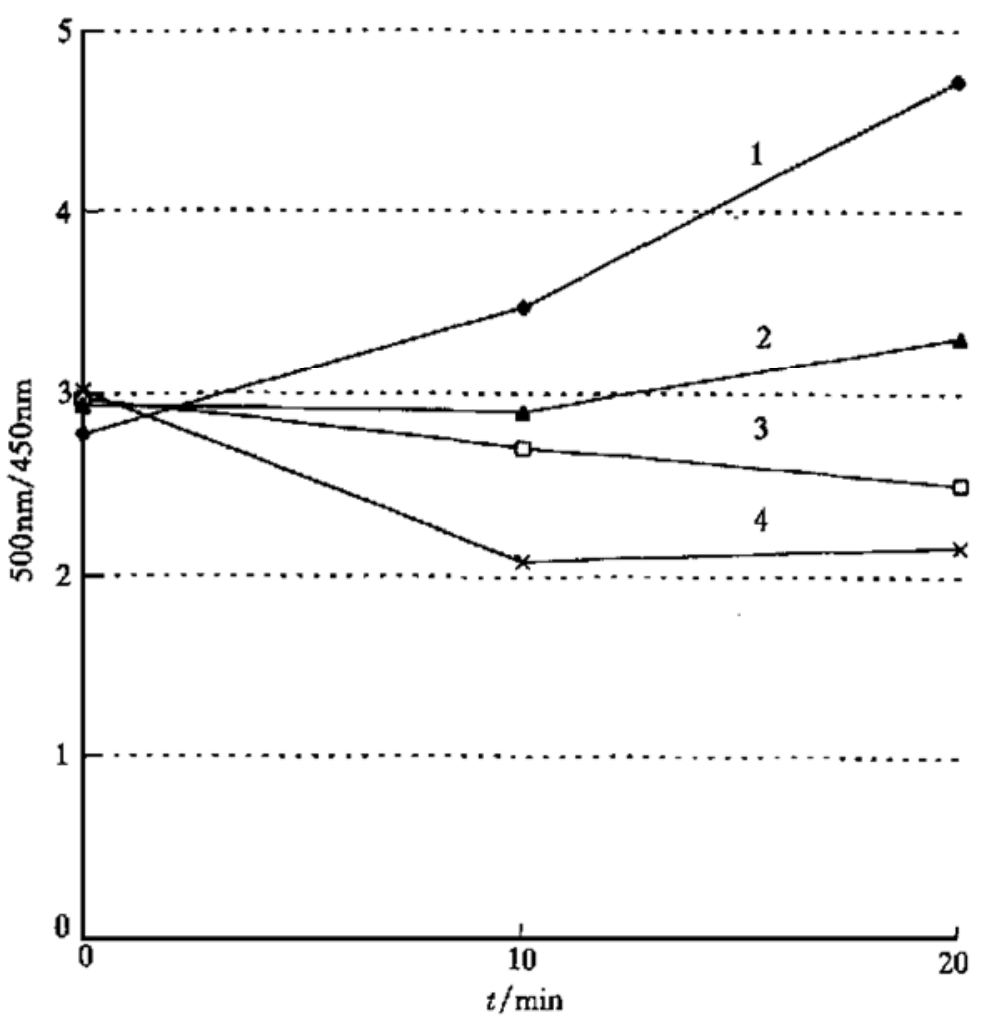

图 1 M_CSF_R 对 J6_1 细胞胞内 $\mathrm{pH}$ 的作用 1 一对照, $2-0.08 \mathrm{U}$ M_CSF_R, 3-20\% 胎牛血清, $4 \longrightarrow 0.16$ U M_CSF_R 有抑制作用, 表明 M_CSF_R 的糖基在
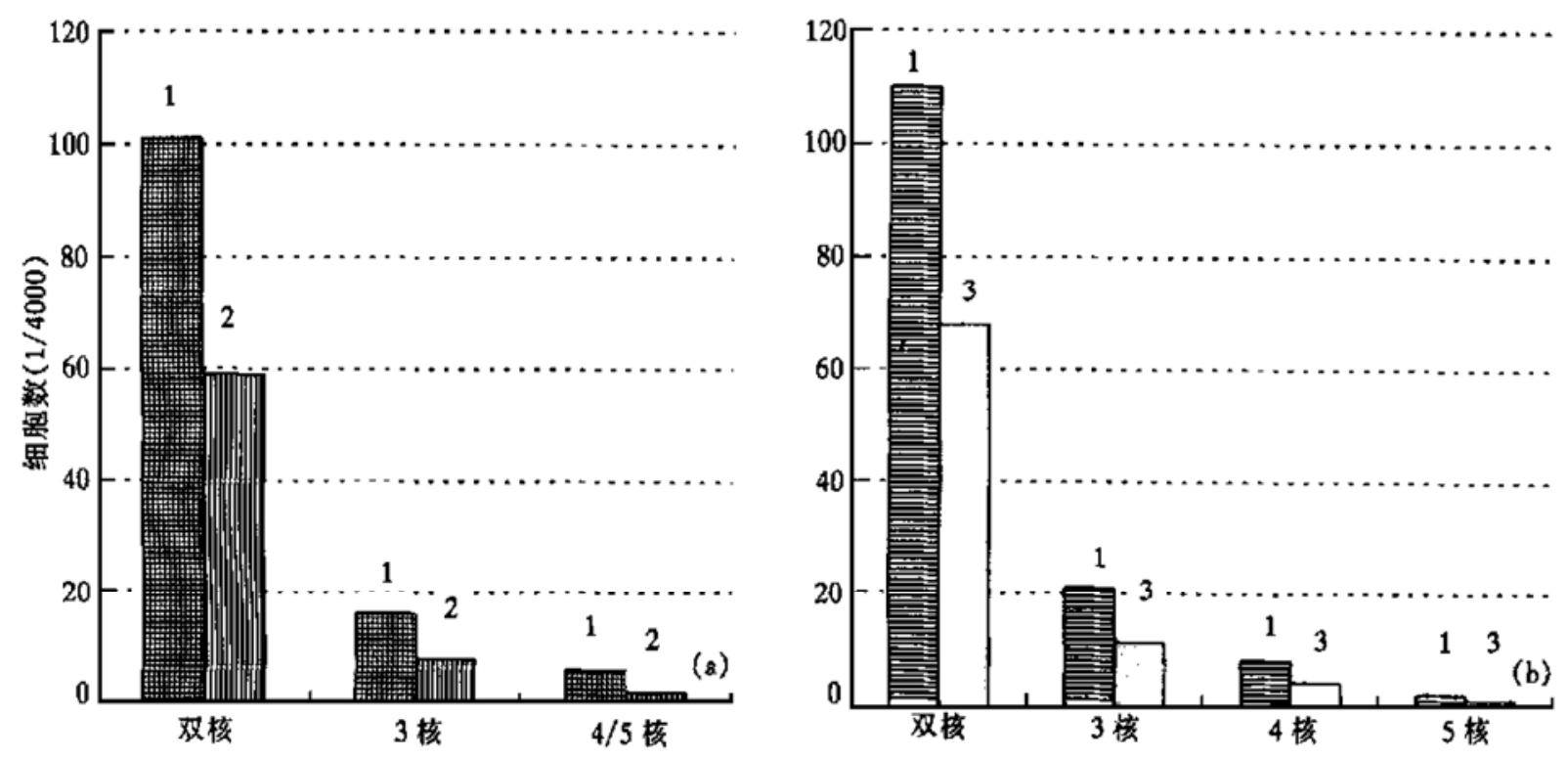

图 2 M_CSF 受体及抗 M_CSF 单抗对 J6_1 细胞多核细胞比例的作用 1 一对照, 2-

与 m_M_CSF 作用中不起重要作用.

本文结果表明 m_M_CSF 在 J6_1 细胞上有受体作用. U emura 等人曾证明 m_M_CSF 与其受 体有粘附分子样作用 ${ }^{8]}$. 粘附分子间以受体和反向受体( counter_recepor) 相互作用, 传递信息, 在 


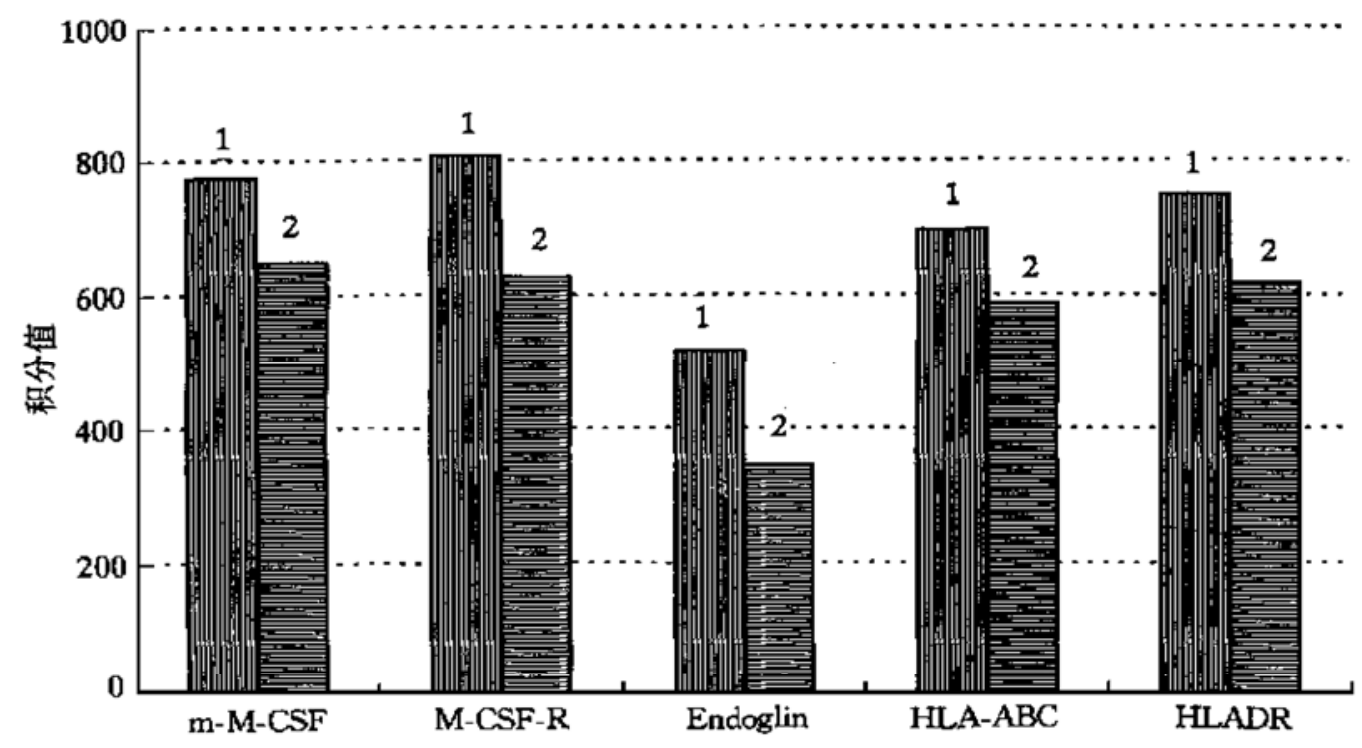

图 3 M_CSF_R 对 J6_1 细胞表面抗原表达的影响 1 一对照, 2 一实验

粘附、细胞识别等基本生物学过程中起重要作用. m_M_CSF 不仅有胞外区还有 36 个氨基酸残 基的胞内区, 从结构上看有受体样结构. 最近 Weiser 等人报道 B 细胞表面免疫球蛋白尽管只有 7 个氨基酸残基构成的胞内区, 但已证明对抗原递呈, 调节免疫应答起重要作用, 它们与胞内 其他分子偶联, 可引发信号传递过程 ${ }^{99}$. m_M_CSF 胞内区的信号传递过程有待研究.

致谢 本工作为国家自然科学基金( 批准号: 39570368) 资助项目.

\section{参 考 文 献}

1 Anklesaria P, Teixido J, Laiho M, et al. Cell_cell adhesion mediated by binding of membrane_anchored transforming growth factor and epidermal grow th factor recptor promotes cell proliferation. Proc Natl Acad Sci USA, 1990, 87: 3 289 3 293

2 吴克复. 张一泉. 齐淑玲. 等. 粒. 单型人白血病细胞系的建立及其细胞生物学性质的研究. 遣传学报. 1980.7: 136 143

3 Wu K F, Rao Q, Zheng G G, et al. Enhancement of J6_1 human leukemic cell proliferation by cell_cell contact: role of an M_ CSF_like membrane_associate grow th factor MAF_J6_1. Leukemia Res, 1994, 18: 843 849

4 Wu K F, Zheng G G, Geng Y Q, et al. Auto_juxtacrine mediated via m_M_CSF/M_CSF_R on human leukemic cells. Cell Res, 1995( suppl), 69 72

5 Wu K F, Rao Q, Zheng G G, et al. Enhancement of J6_1 human leukemic cell proliferation by M AF_J6_1 through a cell_cell contact mechanism (ㄷ. Role of an M_CSF receptor_like membrane protein. Leukemia Res, (in press)

6 耿以琪, 饶 青, 孔 建, 等. 白血病细胞膜相关因子(MAF_J6_1) 单克隆抗体的制备及性质. 中华血液学杂志, 1994, 15: 630 633

7 Song Y H, Yang C L, QiS L, et al. Studies on micromegakaryocytes in myelodysplastic syndromes (MDS). Proc CAMS \& PU M C, 1988, 3: 33 36

8 U emura N. Binding of membrane_anchored macrophage colony_stimulating factor ( M_CSF) to its receptor mediates specific adhesion between stromal cells and M_CSF receptor_bearing hematopoietic cells. Blood, 1993, 82: 2 634 2639

9 Weiser P. Endosomal targeting by the cytoplasmic tial of membrane immunoglobulin. Science, 1997, 276: 407 